\title{
Supplementary Information for Nitroxidative, Nitrosative, and Nitrative Stress: Kinetic Predictions of a Network of Reactive Nitrogen Species Chemistry Under Biological Conditions
}

\author{
Jack R. Lancaster, Jr. \\ University of Alabama Birmingham
}

Supplementary Table 1. Rate constants used in the simulations.

\begin{tabular}{|c|c|c|c|}
\hline $\begin{array}{c}\text { Rate } \\
\text { Constant }\end{array}$ & Reaction & Value & Reference \\
\hline $\mathrm{k}_{1}$ & Formation of $\mathrm{NO}$ & As indicated & -- \\
\hline $\mathrm{k}_{2}$ & Formation of $\mathrm{O}_{2}{ }^{\circ-}$ & As indicated & -- \\
\hline $\mathrm{k}_{3}$ & $\cdot \mathrm{NO}+\mathrm{O}_{2}^{\cdot-} \rightarrow \mathrm{ONOO}^{-}$ & $1.9 \times 10^{10} \mathrm{M}^{-1} \mathrm{~s}^{-1}$ & (1) \\
\hline $\mathrm{k}_{4}$ & $\mathrm{ONOO}^{-}+\mathrm{H}^{+} \rightarrow \cdot \mathrm{NO}_{2}+\cdot \mathrm{OH}$ & $0.232 \mathrm{~s}^{-1}$ & $(2)^{a}$ \\
\hline $\mathrm{k}_{5}$ & $\mathrm{O}_{2}{ }^{--}+\mathrm{NO}_{2} \rightarrow \mathrm{O}_{2} \mathrm{NOO}^{-}$ & $4.5 \times 10^{9} \mathrm{M}^{-1} \mathrm{~s}^{-1}$ & (3) \\
\hline $\mathrm{k}_{6}$ & $\mathrm{O}_{2}^{\cdot-}+\cdot \mathrm{OH} \rightarrow \mathrm{O}_{2}+\mathrm{OH}^{-}$ & $1.0 \times 10^{10} \mathrm{M}^{-1} \mathrm{~s}^{-1}$ & (4) \\
\hline$k_{7}$ & $\cdot \mathrm{NO}+\cdot \mathrm{NO}_{2} \rightarrow \mathrm{N}_{2} \mathrm{O}_{3}$ & $1.1 \times 10^{9} \mathrm{M}^{-1} \mathrm{~s}^{-1}$ & (3) \\
\hline $\mathrm{k}_{-7}$ & $\mathrm{~N}_{2} \mathrm{O}_{3} \rightarrow \cdot \mathrm{NO}+\cdot \mathrm{NO}_{2}$ & $8.1 \times 10^{4} \mathrm{M}^{-1} \mathrm{~s}^{-1}$ & (5) \\
\hline $\mathrm{k}_{8}$ & $\cdot \mathrm{NO}+\cdot \mathrm{OH} \rightarrow \mathrm{HNO}_{2}$ & $1.0 \times 10^{10} \mathrm{M}^{-1} \mathrm{~s}^{-1}$ & (3) \\
\hline $\mathrm{k}_{9}$ & $4 \cdot \mathrm{NO}+\mathrm{O}_{2}+2 \mathrm{H}_{2} \mathrm{O} \rightarrow 4 \mathrm{HNO}_{2}$ & $8 \times 10^{6} \mathrm{M}^{-2} \mathrm{~s}^{-1}$ & (6) \\
\hline $\mathrm{k}_{10}$ & $\begin{array}{c}2 \mathrm{O}_{2}{ }^{\circ-}+2 \mathrm{H}^{+} \rightarrow \mathrm{O}_{2}+\mathrm{H}_{2} \mathrm{O}_{2} \\
\text { (Nonenzymatic) }\end{array}$ & $2.54 \times 10^{5} \mathrm{M}^{-1} \mathrm{~s}^{-1}$ & $(7)$ \\
\hline $\mathrm{k}_{11}$ & $\mathrm{ONOO}^{-}+\mathrm{H}^{+} \rightarrow \mathrm{H}^{+}+\mathrm{NO}_{3}^{-}$ & $0.568 \mathrm{~s}^{-1}(\mathrm{pH} 7.4)$ & $(2)^{\mathrm{a}}$ \\
\hline $\mathrm{k}_{12}$ & Disappearance/ reaction of $\cdot \mathrm{NO}_{2}$ & As indicated & -- \\
\hline $\mathrm{k}_{13}$ & Disappearance/reaction of $\cdot \mathrm{OH}$ & As indicated & -- \\
\hline $\mathrm{k}_{14}$ & $\mathrm{NO}_{2}^{-}+\cdot \mathrm{OH} \rightarrow \cdot \mathrm{NO}_{2}+\mathrm{OH}^{-}$ & $5.3 \times 10^{9} \mathrm{M}^{-1} \mathrm{~s}^{-1}$ & (3) \\
\hline$k_{15}$ & $2 \cdot \mathrm{NO}+\mathrm{O}_{2} \rightarrow 2 \cdot \mathrm{NO}_{2}$ & $2 \times 10^{6} \mathrm{M}^{-2} \mathrm{~s}^{-1}$ & (8) \\
\hline $\mathrm{k}_{16}$ & $\mathrm{ONOO}^{-}+\mathrm{CO}_{2} \rightarrow \mathrm{NO}_{3}^{-}+\mathrm{CO}_{2}$ & $\begin{array}{c}2 \times 10^{4} \mathrm{M}^{-1} \mathrm{~s}^{-1} \\
\left(67 \% \text { of } 3 \times 10^{4} \mathrm{M}^{-1} \mathrm{~s}^{-1}\right)\end{array}$ & (9) \\
\hline $\mathrm{k}_{17}$ & $\mathrm{ONOO}^{-}+\mathrm{CO}_{2} \rightarrow{ }^{\circ} \mathrm{NO}_{2}+\mathrm{CO}_{3}{ }^{-*}$ & $\begin{array}{c}1 \times 10^{4} \mathrm{M}^{-1} \mathrm{~s}^{-1} \\
\left(33 \% \text { of } 3 \times 10^{4} \mathrm{M}^{-1} \mathrm{~s}^{-1}\right)\end{array}$ & (9) \\
\hline $\mathrm{k}_{18}$ & $\mathrm{CO}_{3}^{\cdot-}+\mathrm{RH} \rightarrow \mathrm{HCO}_{3}^{-}+\mathrm{R}^{\cdot}$ & $4 \times 10^{5} M^{-1} s^{-1}$ & (10) \\
\hline $\mathrm{k}_{19}$ & $\cdot \mathrm{NO}_{2}+\mathrm{RH} \rightarrow \mathrm{NO}_{2}^{-}+\mathrm{R}^{\cdot}+\mathrm{H}^{+}$ & $3.2 \times 10^{5} \mathrm{M}^{-1} \mathrm{~s}^{-1}$ & (11) \\
\hline $\mathrm{k}_{20}$ & $\mathrm{R} \cdot+{ }^{*} \mathrm{NO} \rightarrow \mathrm{RNO}$ & $1 \times 10^{10} \mathrm{M}^{-1} \mathrm{~s}^{-1}$ & Assumed \\
\hline $\mathrm{k}_{21}$ & $\mathrm{CO}_{3}{ }^{\circ}+\mathrm{O}_{2}{ }^{--}+\mathrm{H}^{+} \rightarrow \mathrm{HCO}_{3}^{-}+\mathrm{O}_{2}$ & $4 \times 10^{8} \mathrm{M}^{-1} \mathrm{~s}^{-1}$ & (12) \\
\hline $\mathrm{k}_{22}$ & $\begin{array}{c}\mathrm{CO}_{3}^{\cdot^{-}}+\stackrel{ }{\cdot} \mathrm{NO}+\mathrm{OH}^{-} \rightarrow \mathrm{HCO}_{3}^{-}+ \\
\mathrm{NO}_{2}^{-}\end{array}$ & $3.5 \times 10^{9} \mathrm{M}^{-1} \mathrm{~s}^{-1}$ & (13) \\
\hline$k_{23}$ & $\mathrm{~N}_{2} \mathrm{O}_{3}+\mathrm{RH} \rightarrow \mathrm{RNO}+\mathrm{H}^{+}+\mathrm{NO}_{2}^{-}$ & $1.8 \times 10^{8} \mathrm{M}^{-1} \mathrm{~s}^{-1}$ & (5) \\
\hline $\mathrm{k}_{24}$ & $\mathrm{~N}_{2} \mathrm{O}_{3}+\mathrm{H}_{2} \mathrm{O} \rightarrow 2 \mathrm{H}^{+}+2 \mathrm{NO}_{2}^{-}$ & $2.0 \times 10^{3}+10^{8}\left[\mathrm{OH}^{-}\right] \mathrm{s}^{-1}$ & (14) \\
\hline
\end{tabular}




\begin{tabular}{|c|c|c|c|}
\hline $\mathrm{k}_{25}$ & $\cdot \mathrm{NO}_{2}+\mathrm{Tyr} \rightarrow \mathrm{NO}_{2}^{-}+\mathrm{TyrO} \cdot$ & $3.2 \times 10^{5} \mathrm{M}^{-1} \mathrm{~s}^{-1}$ & (11) \\
\hline $\mathrm{k}_{26}$ & $\mathrm{CO}_{3}{ }^{-}+\mathrm{Tyr} \rightarrow \mathrm{HCO}_{3}^{-}$TyrO & $4.5 \times 10^{7} \mathrm{M}^{-1} \mathrm{~s}^{-1}$ & (15) \\
\hline $\mathrm{k}_{27}$ & $\cdot \mathrm{NO}+\mathrm{TyrO} \cdot \rightarrow$ Tyr-ONO & $1 \times 10^{9} \mathrm{M}^{-1} \mathrm{~s}^{-1}$ & (15) \\
\hline $\mathrm{k}_{-27}$ & Tyr-ONO $\rightarrow \cdot \mathrm{NO}^{+}$TyrO & $1 \times 10^{3} \mathrm{~s}^{-1}$ & (15) \\
\hline $\mathrm{k}_{28}$ & Tyr-ONO $\rightarrow$ Products 1 & $0.5 \mathrm{~s}^{-1}$ & See Text \\
\hline $\mathrm{k}_{29}$ & $\mathrm{O}_{2}{ }^{--}+$TyrO $\rightarrow$ Products 2 & $1.5 \times 10^{9} \mathrm{M}^{-1} \mathrm{~s}^{-1}$ & (15) \\
\hline $\mathrm{k}_{30}$ & $2 \mathrm{TyrO} \cdot \rightarrow$ diTyr & $\begin{array}{c}8.05 \times 10^{7} \mathrm{M}^{-1} \mathrm{~s}^{-1} \\
\left(0.35 \times 2.3 \times 10^{8} \mathrm{M}^{-1} \mathrm{~s}^{-1}\right)\end{array}$ & (16) \\
\hline $\mathrm{k}_{31}$ & $\cdot \mathrm{NO}_{2}+\mathrm{TyrO} \cdot \rightarrow$ Products 3 & $1.7 \times 10^{9} \mathrm{M}^{-1} \mathrm{~s}^{-1}$ & (15) \\
\hline $\mathrm{k}_{32}$ & $\cdot \mathrm{NO}_{2}+\mathrm{TyrO} \cdot \rightarrow \mathrm{NO}_{2}-\mathrm{Tyr}$ & $1.3 \times 10^{9} \mathrm{M}^{-1} \mathrm{~s}^{-1}$ & (15) \\
\hline $\mathrm{k}_{33}$ & $\mathrm{ONOO}^{-}+\mathrm{GSH} \rightarrow \mathrm{NO}_{2}^{-}+\mathrm{GSOH}^{-}$ & $6.6 \times 10^{2} \mathrm{M}^{-1} \mathrm{~s}^{-1}$ & $(17)$ \\
\hline $\mathrm{k}_{34}$ & $\begin{array}{c}\mathrm{N}_{2} \mathrm{O}_{3}+\mathrm{GSH} \rightarrow \mathrm{H}^{+}+\mathrm{NO}_{2}^{-}+ \\
\text {GSNO }\end{array}$ & $6.6 \times 10^{7} \mathrm{M}^{-1} \mathrm{~s}^{-1}$ & $(18)$ \\
\hline $\mathrm{k}_{35}$ & $\cdot \mathrm{NO}_{2}+\mathrm{GSH} \rightarrow \mathrm{NO}_{2}^{-}+\mathrm{H}^{+}+\mathrm{GS}^{\cdot}$ & $2 \times 10^{7} \mathrm{M}^{-1} \mathrm{~s}^{-1}$ & (19) \\
\hline $\mathrm{k}_{36}$ & $\mathrm{CO}_{3}{ }^{--}+\mathrm{GSH} \rightarrow \mathrm{HCO}_{3}^{-}+\mathrm{GS}^{-}$ & $5.3 \times 10^{6} \mathrm{M}^{-1} \mathrm{~s}^{-1}$ & (20) \\
\hline $\mathrm{k}_{37}$ & $\cdot \mathrm{NO}+\mathrm{GS} \cdot \rightarrow \mathrm{GSNO}$ & $3 \times 10^{9} \mathrm{M}^{-1} \mathrm{~s}^{-1}$ & (21) \\
\hline $\mathrm{k}_{38}$ & GS $^{*}+$ GS $^{-} \rightarrow$ GSSG $^{-}$ & $0.016 \times 6 \times 10^{8} \mathrm{M}^{-1} \mathrm{~s}^{-1}$ & $(22)^{b}$ \\
\hline $\mathrm{k}_{-38}$ & $\mathrm{GSSG}^{*} \rightarrow \mathrm{GS}^{\cdot}+\mathrm{GS}^{-}$ & $1.6 \times 10^{5} \mathrm{~s}^{-1}$ & (22) \\
\hline $\mathrm{k}_{39}$ & $\mathrm{GSSG}^{-}+\mathrm{O}_{2} \rightarrow \mathrm{GSSG}+\mathrm{O}_{2}^{0^{-}}$ & $5 \times 10^{9} \mathrm{M}^{-1} \mathrm{~s}^{-1}$ & (22) \\
\hline $\mathrm{k}_{40}$ & $2 \mathrm{GS} \cdot \rightarrow \mathrm{GSSG}$ & $1.5 \times 10^{9} \mathrm{M}^{-1} \mathrm{~s}^{-1}$ & $(23)$ \\
\hline $\mathrm{k}_{41}$ & $\cdot \mathrm{NO}_{2}+\mathrm{GS} \cdot \rightarrow \mathrm{GSNO}_{2}$ & $3 \times 10^{9} \mathrm{M}^{-1} \mathrm{~s}^{-1}$ & $(24)$ \\
\hline $\mathrm{k}_{42}$ & GS + GSNO $\rightarrow$ GSSG $+\cdot{ }^{\cdot} O$ & $1.7 \times 10^{9} \mathrm{M}^{-1} \mathrm{~s}^{-1}$ & $(25)$ \\
\hline $\mathrm{k}_{43}$ & $\mathrm{GS}^{\cdot}+\mathrm{O}_{2} \rightarrow \mathrm{GSOO} \cdot$ & $2 \times 10^{9} \mathrm{M}^{-1} \mathrm{~s}^{-1}$ & (22) \\
\hline $\mathrm{k}-43$ & $\mathrm{GSOO}^{*} \rightarrow \mathrm{GS}^{\bullet}+\mathrm{O}_{2}$ & $6 \times 10^{5} \mathrm{~s}^{-1}$ & (22) \\
\hline $\mathrm{k}_{44}$ & $\begin{array}{c}\mathrm{GSOO} \cdot \mathrm{GSNO} \rightarrow \mathrm{GSSG}+\mathrm{O}_{2} \\
+\cdot \mathrm{NO}\end{array}$ & $3.8 \times 10^{8} \mathrm{M}^{-1} \mathrm{~s}^{-1}$ & $(25)$ \\
\hline $\mathrm{k}_{45}$ & $\mathrm{GSOO}^{\bullet}+{ }^{\cdot \mathrm{NO}_{2}} \rightarrow \mathrm{GSOONO}_{2}$ & $1 \times 10^{9} \mathrm{M}^{-1} \mathrm{~s}^{-1}$ & (21) \\
\hline $\mathrm{k}_{-45}$ & $\mathrm{GSOONO}_{2} \rightarrow \mathrm{GSOO}^{\cdot}+\cdot^{\cdot \mathrm{NO}_{2}}$ & $0.75 \mathrm{~s}^{-1}$ & $(21)$ \\
\hline $\mathrm{k}_{46}$ & $2 \mathrm{GSOO} \rightarrow x \mathrm{O}_{2}{ }^{\cdot-}+$ Products 4 & $\begin{array}{c}4 \times 10^{8} \mathrm{M}^{-1} \mathrm{~s}^{-1} \\
x=0.56\end{array}$ & $x=0.56(21)$ \\
\hline $\mathrm{k}_{47}$ & $\mathrm{GSOO}^{\bullet}+{ }^{\cdot} \mathrm{NO} \rightarrow[\mathrm{GSOONO}]$ & $3 \times 10^{9} \mathrm{M}^{-1} \mathrm{~s}^{-1}$ & $(21)\}$ \\
\hline $\mathrm{k}_{48}$ & $\underset{\text { GSOO* }}{\text { GSH }} \rightarrow\left[\right.$ GSOH $\left.^{\circ}\right]+$ & $2 \times 10^{6} \mathrm{M}^{-1} \mathrm{~s}^{-1}$ & (26) \\
\hline $\mathrm{k}_{49}$ & $\cdot \mathrm{NO} \rightarrow$ & As Indicated & \\
\hline $\mathrm{k}_{50}$ & $\begin{array}{c}2 \mathrm{O}_{2}{ }^{--}+2 \mathrm{H}^{+} \rightarrow \mathrm{O}_{2}+\mathrm{H}_{2} \mathrm{O}_{2} \\
(\text { SOD Catalyzed) }\end{array}$ & $1 \times 10^{9} \mathrm{M}^{-1} \mathrm{~s}^{-1}$ & $(27)$ \\
\hline $\mathrm{k}_{51}$ & $\mathrm{GSH}+\mathrm{TyrO} \cdot \rightarrow \mathrm{GS} \cdot+\mathrm{Tyr}$ & $3.5 \times 10^{5} \mathrm{M}^{-1} \mathrm{~s}^{-1}$ & $(28)$ \\
\hline $\mathrm{k}_{-51}$ & $\mathrm{GS}^{\bullet}+$ Tyr $\rightarrow$ GSH + TyrO & $3.5 \times 10^{5} \mathrm{M}^{-1} \mathrm{~s}^{-1}$ & (28) \\
\hline & \multicolumn{3}{|c|}{$\begin{array}{l}\text { The overall rate constant for ONOO disappearance is assumed to be } 0.8 \mathrm{~s}^{-1} \text {, } \\
\text { which is distributed between isomerization to form nitrate }\left(71 \%, \mathrm{k}_{11}=0.568 \mathrm{~s}^{-1}\right) \\
\text { and homolysis to produce }{ }^{\circ} \mathrm{NO}_{2} \text { and } \cdot \mathrm{OH}\left(29 \%, \mathrm{k}_{4}=0.232 \mathrm{~s}^{-1}\right)(2) \text {. } \\
{ }^{\mathrm{b}} \mathrm{pH} 7.4\end{array}$} \\
\hline
\end{tabular}


Supplementary Table 2. Key to Output Columns for Program RNSim1.

Columns 2-39 are instantaneous concentrations of

reactants/intermediates/products. Columns 40-42 are calculations to check for

mass balance (the values should be very close to 1). Columns $43-83$ are

instantaneous rates of reaction for each reaction.

\begin{tabular}{|c|c|c|c|}
\hline Column & Identity & Column & Identity \\
\hline 1 & Time & 22 & $\begin{array}{l}\text { [GSNO] } \\
\text { From k }{ }_{34}\end{array}$ \\
\hline 2 & {$[\mathrm{NO}]_{\mathrm{t}}$} & 23 & {$\left[\mathrm{GS}^{\circ}\right]$} \\
\hline 3 & {$[\mathrm{NO}]_{\text {Total }}$} & 24 & $\begin{array}{l}\text { [GSNO] } \\
\text { From } k_{37}\end{array}$ \\
\hline 4 & {$\left[\mathrm{O}_{2}^{\cdot-}\right]$} & 25 & $\begin{array}{l}\text { [GSSG] } \\
\text { From k } k_{40}\end{array}$ \\
\hline 5 & [ONOO-] & 26 & {$\left[\mathrm{GSSG}^{\circ}{ }^{-}\right]$} \\
\hline 6 & {$\left[{ }^{\bullet} \mathrm{NO}_{2}\right]$} & 27 & $\begin{array}{l}\text { [GSSG] } \\
\text { From } k_{39}\end{array}$ \\
\hline 7 & {$\left[\mathrm{CO}_{3}{ }^{--}\right]$} & 28 & $\begin{array}{l}\text { [GSSG] } \\
\text { From k }\end{array}$ \\
\hline 8 & {$\left[\mathrm{~N}_{2} \mathrm{O}_{3}\right]$} & 29 & $\begin{array}{l}{[G S S G]} \\
\text { From k } k_{44}\end{array}$ \\
\hline 9 & {$\left[\mathrm{NO}_{2}^{-}\right]$} & 30 & {$\left[\mathrm{GSOO}^{\circ}\right]$} \\
\hline 10 & {$\left[\mathrm{NO}_{3}^{-}\right]$} & 31 & {$\left[\mathrm{GSOONO}_{2}\right]$} \\
\hline 11 & [Tyr] & 32 & [Products4] \\
\hline 12 & {$\left[\mathrm{TyrO}^{\bullet}\right]$} & 33 & [Products5] \\
\hline 13 & [ONO-Tyr] & 34 & {$\left[\mathrm{GSONO}_{2}\right]$} \\
\hline 14 & [Products1] & 35 & {$\left[\mathrm{GSNO}_{2}\right]$} \\
\hline 15 & [Products2] & 36 & {$[\mathrm{GSOH}]_{\text {Total }}$} \\
\hline 16 & [diTyr] & 37 & {$[\mathrm{GSNO}]_{\text {Total }}$} \\
\hline 17 & [Products3] & 38 & {$[G S S G]_{\text {Total }}$} \\
\hline 18 & {$\left[\mathrm{NO}_{2} \mathrm{Tyr}\right]$} & 39 & {$\left[\mathrm{O}_{2}\right]$} \\
\hline 19 & {$[\mathrm{GSH}]$} & 40 & {$[\mathrm{NOx}]_{\text {Total }} / \mathrm{NO}_{\text {Total }}$} \\
\hline 20 & $\begin{array}{l}{[\mathrm{GSOH}]} \\
\text { From } \mathrm{k}_{33}\end{array}$ & 41 & {$[\mathrm{GSX}]_{\text {Total }} / \mathrm{GSH}_{\text {Total }}$} \\
\hline 21 & $\begin{array}{l}{[\mathrm{GSOH}]} \\
\text { From } \mathrm{k}_{48}\end{array}$ & 42 & {$[\text { Tyrx }]_{\text {Total }} /[\text { Tyr }]_{\text {Total }}$} \\
\hline
\end{tabular}




\begin{tabular}{|c|c|c|c|}
\hline 43 & $\mathrm{NO}+\mathrm{O}_{2}^{-} \stackrel{\mathrm{K}_{3}}{\longrightarrow} \mathrm{ONOO}$ & 66 & $\mathrm{GSH}+\mathrm{NO}_{2} \stackrel{\mathrm{K}_{35}}{\longrightarrow} \mathrm{GS}+\mathrm{NO}_{2}$ \\
\hline 44 & $\mathrm{ONOO}+\mathrm{CO}_{2} \stackrel{\mathrm{K}_{17}}{\longrightarrow} \mathrm{CO}_{3}^{-\cdots}+\mathrm{NO}_{2}$ & 67 & $\mathrm{GSH}+\mathrm{CO}_{3}^{\cdot} \stackrel{\mathrm{K}_{36}}{\rightarrow \mathrm{GS}}+\mathrm{HCO}_{3}$ \\
\hline 45 & 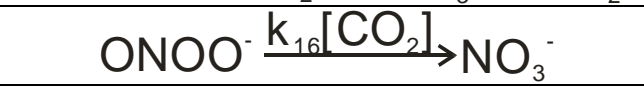 & 68 & $\mathrm{GS} \cdot+\mathrm{NO} \stackrel{\mathrm{k}_{37}}{\rightarrow} \mathrm{GSNO}$ \\
\hline 46 & $\cdot \mathrm{NO}_{2}+\mathrm{O}_{2}^{-\cdot} \stackrel{-\mathrm{K}_{5}}{\longrightarrow} \mathrm{O}_{2} \mathrm{NOO}$ & 69 & $\mathrm{GS}^{-}+\mathrm{GS} \cdot \stackrel{0.016 \times \mathrm{k}_{38}}{\rightarrow} \mathrm{GSSG}^{-}$ \\
\hline 47 & $\mathrm{CO}_{3}^{-}+\mathrm{O}_{2}^{-}+\mathrm{H}^{+} \stackrel{\mathrm{K}_{21}}{\longrightarrow} \mathrm{HCO}_{3}+\mathrm{O}_{2}$ & 70 & GSSG $^{-\mathrm{k}_{-38}} \rightarrow \mathrm{GS}^{-}+\mathrm{GS}$ \\
\hline 48 & $\cdot \mathrm{NO}_{2}+\mathrm{NO}^{\mathrm{K}_{7}} \longrightarrow \mathrm{N}_{2} \mathrm{O}_{3}$ & 71 & $\mathrm{esc}+\mathrm{C} \longrightarrow \mathrm{CaC}+\mathrm{e}$ \\
\hline 49 & $\mathrm{~N}_{2} \mathrm{O}_{3} \stackrel{\mathrm{k}_{-7}}{\rightarrow} \cdot \mathrm{NO}_{2}+\mathrm{NO}$ & 72 & $2 \mathrm{GS} \cdot \stackrel{\mathrm{k}_{40}}{\rightarrow} \mathrm{GSSG}$ \\
\hline 50 & $\mathrm{~N}_{2} \mathrm{O}_{3}+\mathrm{H}_{2} \mathrm{O} \stackrel{\mathrm{K}_{24}}{\longrightarrow} 2 \mathrm{HNO}_{2}$ & 73 & $\mathrm{GS} \cdot+\mathrm{NO}_{2} \stackrel{\mathrm{k}_{41}}{\rightarrow} \mathrm{GSNO}_{2}$ \\
\hline 51 & $\mathrm{CO}_{3}^{-}+\mathrm{NO}+\mathrm{OH}^{-\mathrm{K}_{22}} \mathrm{HCO}_{3}^{-}+\mathrm{NO}_{2}^{-}$ & 74 & $\mathrm{GS}+\mathrm{O}_{2} \mathrm{~K}_{43}>\mathrm{GSOO}$ \\
\hline 52 & $2 \cdot \mathrm{NO}+\mathrm{O}_{2} \stackrel{\mathrm{K}_{15}}{\longrightarrow} 2 \cdot \mathrm{NO}_{2}$ & 75 & $\mathrm{GSOO} \cdot \stackrel{\mathrm{k}_{-43}}{\longrightarrow} \mathrm{GS} \cdot+\mathrm{O}_{2}$ \\
\hline 53 & $2 \mathrm{O}_{2}^{\prime-}+2 \mathrm{H}^{+} \stackrel{\mathrm{K}_{10}}{\rightarrow} \mathrm{H}_{2} \mathrm{O}_{2}+\mathrm{O}_{2}$ & 76 & $\mathrm{GSOO}+\cdot \mathrm{NO}_{2} \stackrel{\mathrm{K}_{45}}{\rightarrow} \mathrm{GSOONO}_{2}$ \\
\hline 54 & $\mathrm{CO}_{3}^{-}+\mathrm{NO}_{2}+\mathrm{H}^{+} \stackrel{\mathrm{K}_{14}}{\longrightarrow} \mathrm{HCO}_{3}+\mathrm{NO}_{2}$ & 77 & $\mathrm{GSOONO}_{2} \stackrel{\mathrm{K}_{45}}{\longrightarrow} \mathrm{GSOO}+\mathrm{NO}_{2}$ \\
\hline 55 & $\mathrm{Tyr}+\mathrm{NO}_{2} \mathrm{~K}_{25} \mathrm{TyrO}+\mathrm{NO}_{2}^{-}$ & 78 & 2GSOO $\cdot \stackrel{\mathrm{K}_{46}}{\longrightarrow} \mathrm{xO}_{2}+$ Products 4 \\
\hline 56 & $\mathrm{CO}_{3}^{-}+\mathrm{Tyr}+\mathrm{H}^{+\mathrm{k}_{26}} \mathrm{TyrO}+\mathrm{HCO}_{3}^{-}$ & 79 & $\mathrm{GSOO}+\cdot \mathrm{NO} \stackrel{\mathrm{K}_{47}}{\longrightarrow}[\mathrm{GSOONO}]$ \\
\hline 57 & $\mathrm{TyrO}+\cdot \mathrm{NO} \stackrel{\mathrm{K}_{27}}{\longrightarrow} \mathrm{ONO}-\mathrm{Tyr}$ & 80 & $\mathrm{GSOO}+\mathrm{GSH} \stackrel{\mathrm{K}_{48}}{\rightarrow} \mathrm{GSOH}+$ Products $5+\mathrm{O}_{2}$ \\
\hline 58 & ONO-Tyr $\stackrel{\mathrm{k}_{22}}{\mathrm{TyO}}+\cdot+\mathrm{NO}$ & 81 &  \\
\hline 59 & ONO-Tyr $\stackrel{\mathrm{k}_{28}}{\rightarrow}$ Products 1 & 82 & 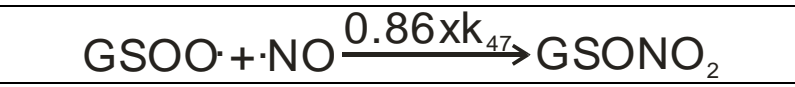 \\
\hline 60 & TyrO $+\mathrm{O}_{2}^{-\mathrm{k}_{29}}$ Products2 & 83 &. $\mathrm{NO} \stackrel{\mathrm{K}_{49} \rightarrow}{\longrightarrow}$ \\
\hline 61 & $2 \mathrm{TyrO} \cdot \stackrel{\mathrm{k}_{30}}{\rightarrow} \mathrm{diTyr}$ & 84 & $\mathrm{GS}+\mathrm{GSNO} \stackrel{\mathrm{k}_{42}}{\longrightarrow} \mathrm{GSSG}+\cdot \mathrm{NO}$ \\
\hline 62 & 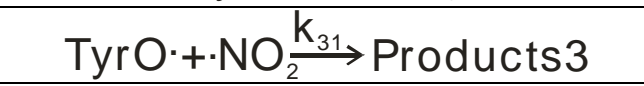 & 85 & 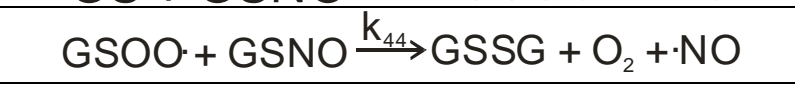 \\
\hline 63 & $\mathrm{TyrO}+\cdot \mathrm{NO}_{2} \underset{\mathrm{K}_{32}}{\longrightarrow} \mathrm{NO}_{2} \mathrm{Tyr}$ & 86 & $\mathrm{O}_{2}^{-}+\mathrm{H}^{+} \stackrel{\mathrm{k}_{50} \mathrm{SOD}}{\longrightarrow} 1 / 2 \mathrm{H}_{2} \mathrm{O}_{2}+1 / 2 \mathrm{O}_{2}$ \\
\hline 64 & $\mathrm{GSH}+\mathrm{ONOO} \stackrel{\mathrm{k}_{33}}{\longrightarrow} \mathrm{GSOH}+\mathrm{NO}_{2}$ & 87 & $\mathrm{GSH}+\mathrm{TyrO} \cdot \stackrel{\mathrm{k}_{51}}{\longrightarrow} \mathrm{GS} \cdot+\mathrm{Tyr}$ \\
\hline 65 & $\mathrm{GSH}+\mathrm{N}_{2} \mathrm{O}_{3} \stackrel{\mathrm{K}_{34}}{\rightarrow} \mathrm{GSNO}+\mathrm{HNO}_{2}$ & 88 & GS. + Tyr $\stackrel{k_{-51}}{\longrightarrow}$ GSH + TyrO. \\
\hline
\end{tabular}




\section{References}

1. Kissner, R., Nauser, T., Bugnon, P., Lye, P. G., and Koppenol, W. H. (1997)Formation and properties of peroxynitrite as studied by laser flash photolysis, high-pressure stopped-flow technique, and pulse radiolysis Chem. Res. Toxicol. 10, 1285-1292.

2. Coddington, J. W., Hurst, J. K., and Lymar, S. V. (1999)Hydroxyl radical Formation During Peroxynitrous Acid Decomposition J. Am. Chem. Soc. $121,2443$.

3. Goldstein, S. and Czapski, G. (2000)Reactivity of peroxynitrite versus simultaneous generation of $\left(^{*}\right) \mathrm{NO}$ and $\mathrm{O}(2)\left({ }^{*}\right)(-)$ toward $\mathrm{NADH}$ Chem. Res. Toxicol. 13, 736-741.

4. Jourd'heuil, D., Jourd'heuil, F. L., Kutchukian, P. S., Musah, R. A., Wink, D. A., and Grisham, M. B. (2001)Reaction of superoxide and nitric oxide with peroxynitrite. Implications for peroxynitrite-mediated oxidation reactions in vivo J. Biol. Chem. 276, 28799-28805.

5. Goldstein, S. and Czapski, G. (1996)Mechanism of the Nitrosation of Thiols and Amines by Oxygenated NO Solutions: The Nature of the Nitrosating Intermediates J. Am. Chem. Soc. 118, 3425.

6. Ford, P. C., Wink, D. A., and Stanbury, D. M. (1993)Autoxidation kinetics of aqueous nitric oxide FEBS Lett. 326, 1-3.

7. Behar, D., Czapski, G., Rabani, J., Dirfman, L. M., and Schwarz, H. A. (1970)The Acid Dissociation Constant and Decay Kinetics of the Perhydroxyl Radical J. Phys. Chem. 74, 3209-3213.

8. Czapski, G. and Goldstein, S. (1995)The role of the reactions of .NO with superoxide and oxygen in biological systems: a kinetic approach. Free Rad. Biol. Med. 19, 785-794. 
9. Lymar, S. V. and Hurst, J. K. (1995)Rapid Reaction between Peroxonitrite Ion and Carbon Dioxide: Implications for Biological Activity J. Am. Chem. Soc. $117,8867-8868$.

10. Augusto, O., Bonini, M. G., Amanso, A. M., Linares, E., Santos, C. C., and De Menezes, S. L. (2002)Nitrogen dioxide and carbonate radical anion: two emerging radicals in biology Free Radic. Biol. Med. 32, 841-859.

11. Prutz, W. A., Monig, H., Butler, J., and Land, E. J. (1985)Reactions of nitrogen dioxide in aqueous model systems: oxidation of tyrosine units in peptides and proteins Arch. Biochem. Biophys. 243, 125-134.

12. Behar, D., Czapski, G., and Duchovny, I. (1970)Carbonate Radical in Flash Photolysis and Pulse Radiolysis of Aqueous Carbonate Solutions J. Phys. Chem. 74, 2206-2210.

13. Czapski, G., Holcman, J., and Bielski, B. H. (1994)Reactivity of Nitric Oxide with Simple Short-lived Radicals in Aqueous Solutions J. Am. Chem. Soc. 116, 11465-11469.

14. Treinin, A. and Hayon, E. (1970)Absorption Spectra and Reaction Kinetics of NO2, N2O3, and N2O4 in Aqueous Solution J. Am. Chem. Soc. 92, 5821-5828.

15. Goldstein, S., Czapski, G., Lind, J., and Merenyi, G. (2000)Tyrosine nitration by simultaneous generation of (.)NO and O-(2) under physiological conditions. How the radicals do the job J. Biol. Chem. 275, 3031-3036.

16. Hodges, G. R., Marwaha, J., Paul, T., and Ingold, K. U. (2000)A novel procedure for generating both nitric oxide and superoxide in situ from chemical sources at any chosen mole ratio. First application: tyrosine oxidation and a comparison with preformed peroxynitrite Chem. Res. Toxicol. 13, 1287-1293. 
17. Bonini, M. G. and Augusto, O. (2001)Carbon dioxide stimulates the production of thiyl, sulfinyl, and disulfide radical anion from thiol oxidation by peroxynitrite J. Biol. Chem. 276, 9749-9754.

18. Keshive, M., Singh, S., Wishnok, J. S., Tannenbaum, S. R., and Deen, W. M. (1996)Kinetics of S-nitrosation of thiols in nitric oxide solutions Chem. Res. Toxicol. 9, 988-993.

19. Ford, E., Hughes, M. N., and Wardman, P. (2002)Kinetics of the reactions of nitrogen dioxide with glutathione, cysteine, and uric acid at physiological pH Free Radic. Biol. Med. 32, 1314-1323.

20. Chen, S. N. and Hoffman, M. Z. (1973)Rate constants for the reaction of the carbonate radical with compounds of biochemical interest in neutral aqueous solution Radiat. Res. 56, 40-47.

21. Goldstein, S., Lind, J., and Merenyi, G. (2004)Reaction of Organic Peroxyl Radicals with NO2 and NO in Aqueous Solution: Intermediacy of OrganicPeroxynitrate and Peroxynitrite Species J. Phys. Chem. 108, 1719-1725.

22. Wardman, P. and von Sonntag, C. (1995)Kinetic factors that control the fate of thiyl radicals in cells Methods Enzymol. 251, 31-45.

23. Hoffman, M. Z. and Hayon, E. (1973)Pulse Radiolysis Study of Sulfhydryl Compounds in Aqueous Solution J. Phys. Chem. 77, 990-996.

24. Kirsch, M., Lehnig, M., Korth, H. G., Sustmann, R., and de Groot, H. (2001)Inhibition of peroxynitrite-induced nitration of tyrosine by glutathione in the presence of carbon dioxide through both radical repair and peroxynitrate formation Eur. Chem. J. 7, 3313-3320.

25. Wood, P. D., Mutus, B., and Redmond, R. W. (1996)The Mechanism of Photochemical Release of Nitric Oxide from S-Nitrosoglutathione Photochem. Photobiol. 64, 518-524. 
26. Wardman, P. (1998)Evaluation of the 'Radical Sink' Hypothesis from a Chemical-Kinetic Viewpoint J. Radioanal. Nucl. Chem. 232, 23-27.

27. Radi, R., Denicola, A., Alvarez, B., Ferrer-Sueta, G., and Rubbo, H. (2000) The Biological Chemistry of Peroxynitrite. In Nitric Oxide: Biology and Pathobiology (Ignarro, L. J., Ed.) pp 57-81, Academic Press, San Diego.

28. Quijano, C., Romero, N., and Radi, R. (2005)Tyrosine nitration by superoxide and nitric oxide fluxes in biological systems: modeling the impact of superoxide dismutase and nitric oxide diffusion Free Radic. Biol. Med. 39, 728-741. 